\title{
What Differs on the Enzymatic Acetylation Mechanisms for Arylamines and Arylhydrazines Substrates? A Theoretical Study
}

\author{
Qing-An Qiao, ${ }^{1}$ Xiao-Min Sun, ${ }^{2}$ Jie Jing, ${ }^{1}$ Xin Chen, ${ }^{1}$ Hua-Yang Wang, ${ }^{3}$ Chuan-Lu Yang, ${ }^{3}$ \\ and Zheng-Ting Cai ${ }^{4}$
}

${ }^{1}$ School of Chemistry and Materials Science, Ludong University, Yantai 264025, China

${ }^{2}$ Environment Research Institute, Shandong University, Jinan 250100, China

${ }^{3}$ School of Physics and Electronic Engineering, Ludong University, Yantai 264025, China

${ }^{4}$ Institute of Theoretical Chemistry, Shandong University, Jinan, Shandong 250100, China

Correspondence should be addressed to Qing-An Qiao, qiaoqa@sdu.edu.cn

Received 12 May 2009; Accepted 8 July 2009

Recommended by George Perry

\begin{abstract}
The acetylation mechanisms of several selected typical substrates from experiments, including arylamines and arylhydrazines, are investigated with the density functional theory in this paper. The results indicate that all the transition states are characterized by a four-membered ring structure, and hydralazine (HDZ) is the most potent substrate. The bioactivity for all the compounds is increased in a sequence of $\mathrm{PABA} \approx 4$-AS $<4-\mathrm{MA}<5$-AS $\approx \mathrm{INH}<\mathrm{HDZ}$. The conjunction effect and the delocalization of the lone pairs of $\mathrm{N}$ atom play a key role in the reaction. All the results are consistent with the experimental data.
\end{abstract}

Copyright (C) 2009 Qing-An Qiao et al. This is an open access article distributed under the Creative Commons Attribution License, which permits unrestricted use, distribution, and reproduction in any medium, provided the original work is properly cited.

\section{Introduction}

The arylamine $\mathrm{N}$-acetyltransferases (NATs, EC 2.3.1.5) are phase II metabolism enzymes found in both prokaryotes and eukaryotes [1]. The $\mathrm{N}$-acetylation reaction leads to the detoxification of arylamine xenobiotics and finally directs to electrophilic arylnitrenium ions, which are considered to be responsible for DNA adduct formation $[2,3]$. To Humans, the two functional NAT isozymes, NAT1 and NAT2, show great differences in substrate specificity and tissue distribution in spite of $81 \%$ amino acid sequence identity [4]. The latter, NAT2, is expressed predominantly in liver and intestinal epithelium [5]. Previous studies [2, $6,7]$ have supposed that NATs catalyze an acetyl transfer by a classical ping-pong kinetic mechanism (Scheme 1). Site-directed mutagenesis analysis of human NAT2 and Salmonella typhimurium NAT (StNAT) $[7,8]$ suggested that a cysteine residue in the active site was responsible for mediating the acetylation process. A recent presteady-state and steady-state kinetic studies on p-nitrophenyl acetate (PNPA) and NAT2 $[9,10]$ revealed that the catalytic mechanism of NAT2 might depend on the formation of a thiolate-imidazolium pair. Though the enzyme is found in both eukaryotes and prokaryotes, the endogenous roles of NATs are still unclear [11]. The substrate determination revealed that both arylamines and arylhydrazines could be acetylated by NATs [11-15]. The required steps in the acetyl transfer reaction are composed of the acetyl group transfer from the active site cysteine residue to the substrate, and the removal of one proton from the latter to the former. In this paper, a detailed theoretical study on the behaviors of arylamines and arylhydrazines substrates in acetylation is available, including the properties of their structures, the transition states, the profiles of energies.

\section{Methodologies}

All calculations were performed with the density functional theory (DFT) B3LYP [16-18] method as implemented in Gaussian03 program package [19], which has previously been successfully employed on a number of enzymatic systems [20]. When $6-31 \mathrm{G}^{*}$ and $6-311+\mathrm{G}(3 \mathrm{df}, 2 \mathrm{p}$ ) basis sets were used, the B3LYP hybrid functional was preferred to Hartree-Fock (HF) and MP2 methods [21, 22]. Though it sometimes fails in treatment of dispersion-rich interactions, 


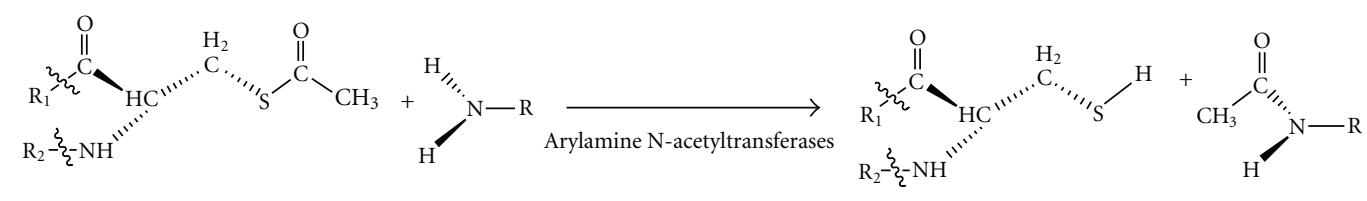

The acetylated active sitecysteine Substrates

Scheme 1: The NATs catalyzed acetyl transfer reaction.
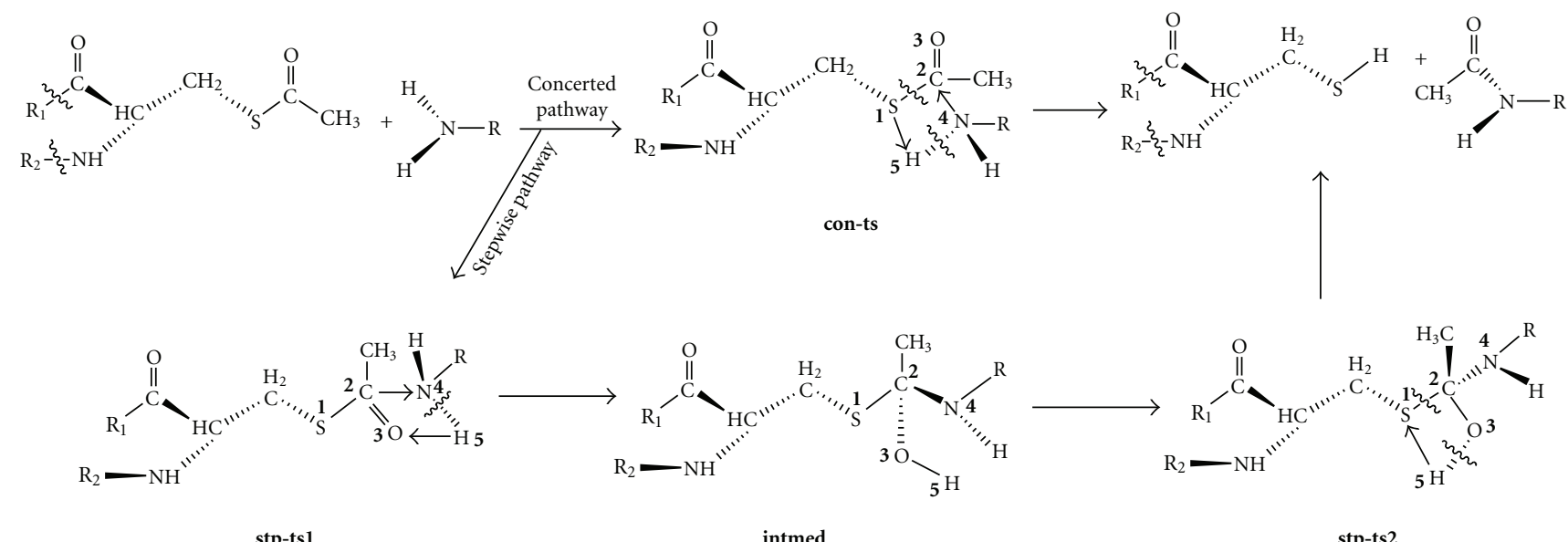

FIGURE 1: The concerted and stepwise pathways for arylamine $\mathrm{N}$-acetyltransferases catalyzed reaction.

the B3LYP method has been successfully applied on many biological systems [23-25].

The geometries of all the reactants, intermediates, and products are optimized at the $\mathrm{B} 3 \mathrm{LYP} / 6-31 \mathrm{G}^{*}$ level of theory. The most stable conformations as well as their energies at every equilibration and transition states have been figured out. Frequency calculations are performed to all the resulting stationary points and each transition states has only one imaginary frequency. Furthermore, MP2/6$311+\mathrm{G}^{* *}$ method is employed on the optimized structures of stationary points to get more accuracy energy profiles. If not specially pointed out, all the following energy analyses refer to the results from MP2/6-311+G**//B3LYP/6-31G (d) calculations.

\section{Results and Discussion}

3.1. The Substrates' Frontier Obitals. Six substrates, $p$ aminobenzoic acid(PABA), 4-methoxylaniline(4-MA), 4aminosalicylate (4-AS), 5-aminosalicylate(5-AS), isoniazid(INZ), hydralazine (HDZ), are selected according the references [11-15], which can be divided into two distinct families: arylamines and arylhydrazines. The energies for frontier obitals (including HOMO-2, HOMO-1, HOMO, LUMO, LUMO+1 and LUMO+2) of all six substrates are listed in Table 1, which are considered playing important roles in bioenzymatic systems [26]. For the arylamines, the HOMO energies are reduced in a sequence of 4-MA > 5-AS > PABA > 4-AS, which indicates the increasing of their nucleophilic reactivity. Among all the six substrates (see Figure 1 in the supplementary materials available online at doi: 10.1155/2009/783035), INZ has the largest energy gap between HOMO and LUMO, which suggests its stability. The Natural Population Analysis (NPA) results showed that the active amino $\mathrm{N}$ atoms for the arylamines family are more electronegative than those of the hydrazines group, which is mainly caused by the conjugation effect.

Though PABA and 4-AS have different substitutions on the $p$ - site of the amino group at the six-membered ring, their bioactivities are nearly the same. An intermolecular $\mathrm{H}$-bond will stabilize the substrate itself with the energy drop about $19-21 \mathrm{~kJ} / \mathrm{mol}$. Both the HOMO and LOMO energies for 5-AS are higher than those of $4-\mathrm{AS}^{\prime} \mathrm{s}$, suggesting that the former is more reactive than the latter.

For the arylhydrazines substrates, the lone pair of the $\mathrm{N}$ atoms at the backbone of the six-membered ring will be delocalized at the whole system so as to enhance its stability. The $E_{(L-H)}$ values of HDZ is 0.0318 a.u. higher than that of INZ's, indicating its higher reactivity than the latter.

3.2. The Different Pathways and Transition States. In principle, all the substrates can react with the active site cysteine residue via a concerted pathway or a stepwise one. To the former, the transition states (see Figures 1 and 2, con-ts) experience a concerted transfer of the hydrogen $\mathrm{H} 5$ to $\mathrm{S} 1$ atom of cysteine and a bond formation between $\mathrm{N} 4$ and $\mathrm{C} 2$ atoms. The broken of old bonds (N4H5 and S1C2) and the formation of new ones (C2N4 and S1H5) take place 


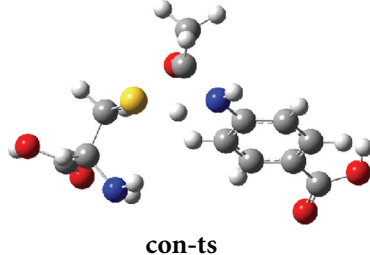

(a)

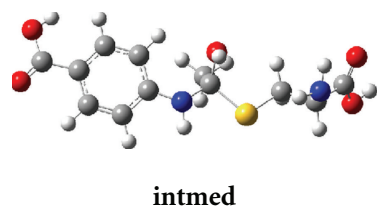

(c)

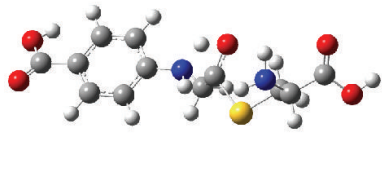

stp-ts1

(b)

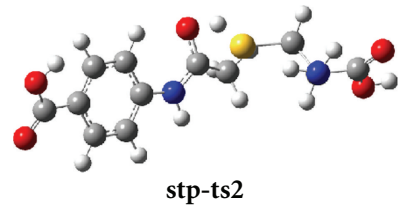

(d)

FIGURE 2: The structure of transition states for both concerted and stepwise pathway of PABA.

TABLE 1: The energies of the frontier orbitals.

\begin{tabular}{lccccccc}
\hline Energy/a.u. & HOMO-2 & HOMO-1 & HOMO & LUMO & LUMO+1 & LUMO+2 & $E_{(L-H)}^{(a)}$ \\
\hline PABA & -0.4469 & -0.3636 & -0.3183 & 0.0536 & 0.0660 & 0.0749 \\
4-MA & -0.4366 & -0.3398 & -0.2890 & 0.0693 & 0.0815 & 0.0863 \\
4-AS & -0.4597 & -0.3443 & -0.3244 & 0.0525 & 0.0627 & 0.3583 \\
5-AS & -0.4419 & -0.3641 & -0.2924 & 0.0611 & 0.0619 & 0.0729 & 0.3769 \\
INZ & -0.4162 & -0.3850 & -0.3484 & 0.0599 & 0.0655 & 0.0787 & 0.3535 \\
HDZ & -0.3822 & -0.3664 & -0.3171 & 0.0590 & 0.0605 & 0.0656 & 0.37683 \\
\hline
\end{tabular}

${ }^{(a)} E_{(L-H)}$ refers to the energy difference between HOMO and LUMO.

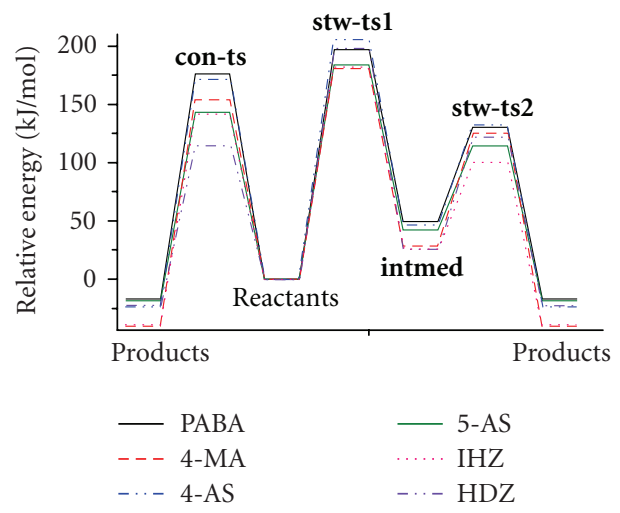

Figure 3: The energy profiles for all substrates.

simultaneously. The reactants and the target products are connected by the only transition state on the potential energy surface (PES). The main structure data of all transition states are listed in Table 1 of the supplementary materials. For the stepwise mechanism, $\mathrm{H} 5$ atom will be firstly transferred to the $\mathrm{O} 3$ atom of the carbonyl group, tending to generate a new bond between $\mathrm{N} 4$ and $\mathrm{C} 2$ atom via transition state stwts1. Then, a thiolester intermediate (intmed) will be formed. Consequently, the second migration of $\mathrm{H} 5$ will take place from the hydroxyl group to S1 atom via stw-ts 2 together with the rupture of S1-C2 bond, which will finally direct to the products.
The results indicate that all the transition states are characterized by a four-membered ring structure which is nearly planar. There are two small angles less than $80^{\circ}$ in every transition state (C2S1H5 and S1C2N4 for con-ts, $\mathrm{C} 2 \mathrm{O} 3 \mathrm{H} 5$ and $\mathrm{C} 2 \mathrm{~N} 4 \mathrm{H} 5$ for stp-ts1, C2S1H5 and S1C2O3 for stp-ts2), which bring great strain to the whole system and make it unstable. Among the six concerted transition states (con-ts) of all the substrates, the properties of the bond $\mathrm{C} 2 \mathrm{~N} 4, \mathrm{~N} 4 \mathrm{H} 5$ and S1H5 are roughly the same for all the substrates, while the interaction between S1 and C2 is one of the determining factors for the concerted step.

Things become different for the stepwise pathways. The hybridization changes of $\mathrm{C} 2$ atom follow a similar tendency $\left(s p^{3} \rightarrow s p^{2} \rightarrow s p^{3}\right)$ for all the substrates during the stepwise acetylating (Table 1 supplementary). The first migration of $\mathrm{H} 5$ will lead to transition state stp-ts1, and then an intermediate named intmed is located on the potential energy surface (PES), which is a local minimum. It is a tetrahedral thiolester intermediate as proposed in previous experimental studies [10]. It is short-lived and a consequent H5 transfer will soon occur via transition state stp-ts2. For the stepwise pathway, the structures of the traction states for different substrates differ very little with others. The $3 \mathrm{D}$ structures of the transition states were listed for PABA (Figure 2), the other ones were similar to those.

3.3. The Energies. The relative energies of all possible pathways for the six substrates are carried out based on the 
energy sum of the reactants taken as zero (Figure 3). From Figure 3, we find that the concerted pathways are favored to the stepwise ones. The energy barriers of concerted transition states (con-ts) are lower than those of the stepwise ones (stp-ts1) at a range of $83.5 \mathrm{~kJ} / \mathrm{mol}$ to $26.9 \mathrm{~kJ} / \mathrm{mol}$ (Table 2 supplementary). The arylhydrazines are better substrates than the arylamines, and HDZ is the most reactive one with the lowest activation energy, which meets the experimental data with good agreement [12]. This conclusion could be also drawn from the structure data analysis (Table 1 supplementary). The enhanced conjunction effect and the delocalization of the nitrogen lone pairs at the backbone stabilized the transition state. The bioactivity for all the substrates is increased in a sequence of $\mathrm{PABA} \approx 4-\mathrm{AS}<$ $4-\mathrm{MA}<5-\mathrm{AS} \approx \mathrm{INH}<\mathrm{HDZ}$.

\section{Conclusions}

The following conclusions can be drawn.

(i) All the substrate can be acetylated via two different pathways: the concerted and the stepwise, and the former is much preferred owing to the lower activation energies.

(ii) From our calculation, arylhydrazines are better substrates than the arylamines, and HDZ is the most reactive one with the lowest activation energy. The bioactivity for all the substrates is increased in a sequence as $\mathrm{PABA} \approx 4-\mathrm{AS}<4-\mathrm{MA}<5-$ $\mathrm{AS} \approx \mathrm{INH}<\mathrm{HDZ}$, which is consistent with the experimental results very well [12].

(iii) The conjunction effect and the delocalized lone pairs play very important roles in acetylation. The enhanced conjunction effect and the increasing numbers of the lone pairs at the six-membered ring will lead to the lower energy barrier.

\section{Acknowledgments}

This work was supported by the National Natural Scientific Foundation of China (no.20603030, no.20873074, and no.10674114), 973 project of the Ministry of Science and Technology of China (no. 2009CB930103), the Natural Scientific Foundation of Shandong Province (no. Q2008B07), and the Foundation for Creative Research Groups of Ludong University (no. 08-CXA001).

\section{References}

[1] P. E. Hanna, "N-acetyltransferases, O-acetyltransferases, and N,O-acetyltransferases: enzymology and bioactivation," Advances in Pharmacology, vol. 27, pp. 401-430, 1994.

[2] P. E. Hanna, "Metabolic activation and detoxification of arylamines," Current Medicinal Chemistry, vol. 3, no. 3, pp. 195-210, 1996.

[3] D. W. Hein, D. M. Grant, and E. Sim, "Update on consensus arylamine N-acetyltransferase gene nomenclature," Pharmacogenetics, vol. 10, no. 4, pp. 291-292, 2000.
[4] D. M. Grant, M. Blum, M. Beer, and U. A. Meyer, "Monomorphic and polymorphic human arylamine $\mathrm{N}$-acetyltransferases: a comparison of liver isozymes and expressed products of two cloned genes," Molecular Pharmacology, vol. 39, no. 2, pp. 184191, 1991.

[5] A. Upton, N. Johnson, J. Sandy, and E. Sim, "Arylamine $\mathrm{N}$-acetyltransferases - of mice, men and microorganisms," Trends in Pharmacological Sciences, vol. 22, no. 3, pp. 140-146, 2001.

[6] J.-M. Dupret and D. M. Grant, "Site-directed mutagenesis of recombinant human arylamine $\mathrm{N}$-acetyltransferase expressed in Escherichia coli. Evidence for direct involvement of Cys68 in the catalytic mechanism of polymorphic human NAT2," Journal of Biological Chemistry, vol. 267, no. 11, pp. 73817385, 1992.

[7] M. Watanabe, T. Sofuni, and T. Nohmi, "Involvement of Cys69 residue in the catalytic mechanism of $\mathrm{N}$-hydroxyarylamine O-acetyltransferase of Salmonella typhimurium. Sequence similarity at the amino acid level suggests a common catalytic mechanism of acetyltransferase for S. typhimurium and higher organisms," Journal of Biological Chemistry, vol. 267, no. 12 , pp. 8429-8436, 1992.

[8] J. C. Sinclair, J. Sandy, R. Delgoda, E. Sim, and M. E. M. Noble, "Structure of arylamine N-acetyltransferase reveals a catalytic triad," Nature Structural Biology, vol. 7, no. 7, pp. 560-564, 2000.

[9] J. Sandy, A. Mushtaq, A. Kawamura, J. Sinclair, E. Sim, and M. Noble, "The structure of arylamine $\mathrm{N}$-acetyltransferase from Mycobacterium smegmatis-an enzyme which inactivates the anti-tubercular drug, isoniazid," Journal of Molecular Biology, vol. 318, no. 4, pp. 1071-1083, 2002.

[10] H. Wang, G. M. Vath, K. J. Gleason, P. E. Hanna, and C. R. Wagner, "Probing the mechanism of hamster arylamine $\mathrm{N}$ acetyltransferase 2 acetylation by active site modification, sitedirected mutagenesis, and pre-steady state and steady state kinetic studies," Biochemistry, vol. 43, no. 25, pp. 8234-8246, 2004.

[11] N. Zhang, L. Liu, F. Liu, C. R. Wagner, P. E. Hanna, and K. J. Walters, "NMR-based model reveals the structural determinants of mammalian arylamine $\mathrm{N}$-acetyltransferase substrate specificity," Journal of Molecular Biology, vol. 363, no. 1, pp. 188-200, 2006.

[12] J. Sandy, S. Holton, E. Fullam, E. Sim, and M. Noble, "Binding of the anti-tubercular drug isoniazid to the arylamine $\mathrm{N}$ acetyltransferase protein from Mycobacterium smegmatis," Protein Science, vol. 14, no. 3, pp. 775-782, 2005.

[13] A. Kawamura, J. Graham, A. Mushtaq, et al., "Eukaryotic arylamine $\mathrm{N}$-acetyltransferase investigation of substrate specificity by high-throughput screening," Biochemical Pharmacology, vol. 69, no. 2, pp. 347-359, 2005.

[14] J.-M. Dupret and F. Rodrigues-Lima, "Structure and regulation of the drug-metabolizing enzymes arylamine $\mathrm{N}$ acetyltransferases," Current Medicinal Chemistry, vol. 12, no. 3, pp. 311-318, 2005.

[15] J.-M. Dupret, J. Dairou, N. Atmane, and F. Rodrigues-Lima, "Inactivation of human arylamine $\mathrm{N}$-acetyltransferase 1 by hydrogen peroxide and peroxynitrite," Methods in Enzymology, vol. 400, pp. 215-229, 2005.

[16] A. D. Becke, "A new mixing of Hartree-Fock and local densityfunctional theories," The Journal of Chemical Physics, vol. 98, no. 2, pp. 1372-1377, 1993.

[17] A. D. Becke, "Density-functional thermochemistry. III. The role of exact exchange," The Journal of Chemical Physics, vol. 98, no. 7, pp. 5648-5652, 1993. 
[18] A. D. Becke, "Current density in exchange-correlation functionals: application to atomic states," Journal of Chemical Physics, vol. 117, no. 15, pp. 6935-6938, 2002.

[19] Gaussian03, Revision B.05, Gaussian, Inc., Pittsburgh Pa, USA, 2003.

[20] P. E. M. Siegbahn and M. R. A. Blomberg, "Transition-metal systems in biochemistry studied by high-accuracy quantum chemical methods," Chemical Reviews, vol. 100, no. 2, pp. 421437, 2000.

[21] P. E. M. Siegbahn, "Modeling aspects of mechanisms for reactions catalyzed by metalloenzymes," Journal of Computational Chemistry, vol. 22, no. 14, pp. 1634-1645, 2001.

[22] C. W. Bauschlicher Jr., "A comparison of the accuracy of different functionals," Chemical Physics Letters, vol. 246, no. 1-2, pp. 40-44, 1995.

[23] L. A. Curtiss, K. Raghavachari, P. C. Redfern, and J. A. Pople, "Assessment of Gaussian-3 and density functional theories for a larger experimental test set," Journal of Chemical Physics, vol. 112, no. 17, pp. 7374-7383, 2000.

[24] R. A. Friesner, "Ab initio quantum chemistry: methodology and applications," Proceedings of the National Academy of Sciences of the United States of America, vol. 102, no. 19, pp. 6648-6653, 2005.

[25] F. Himo, "Quantum chemical modeling of enzyme active sites and reaction mechanisms," Theoretical Chemistry Accounts, vol. 116, no. 1-3, pp. 232-240, 2006.

[26] W. G. Richards, Quantum Pharmacology, Butterworths, London, UK, 2nd edition, 1983. 

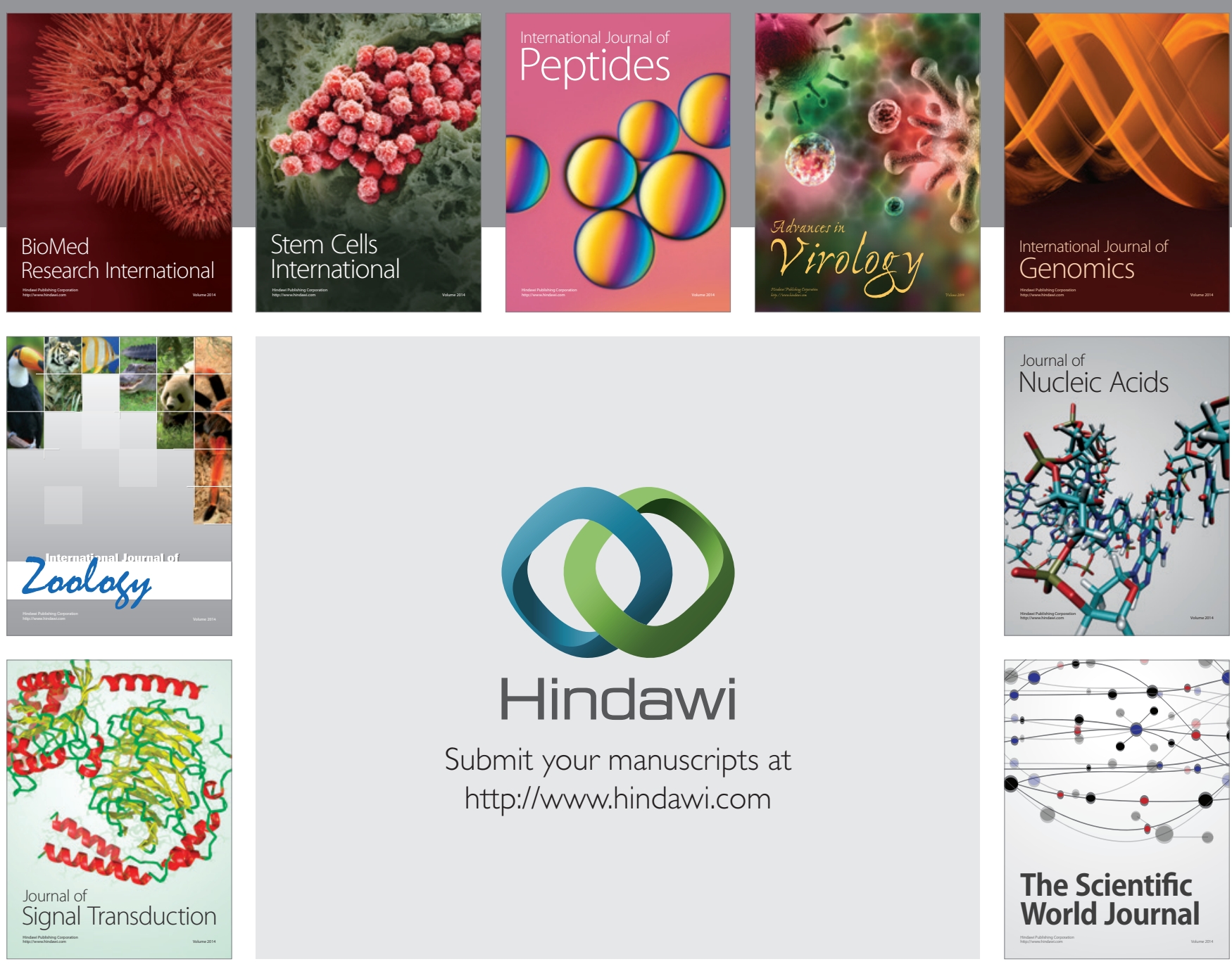

Submit your manuscripts at

http://www.hindawi.com
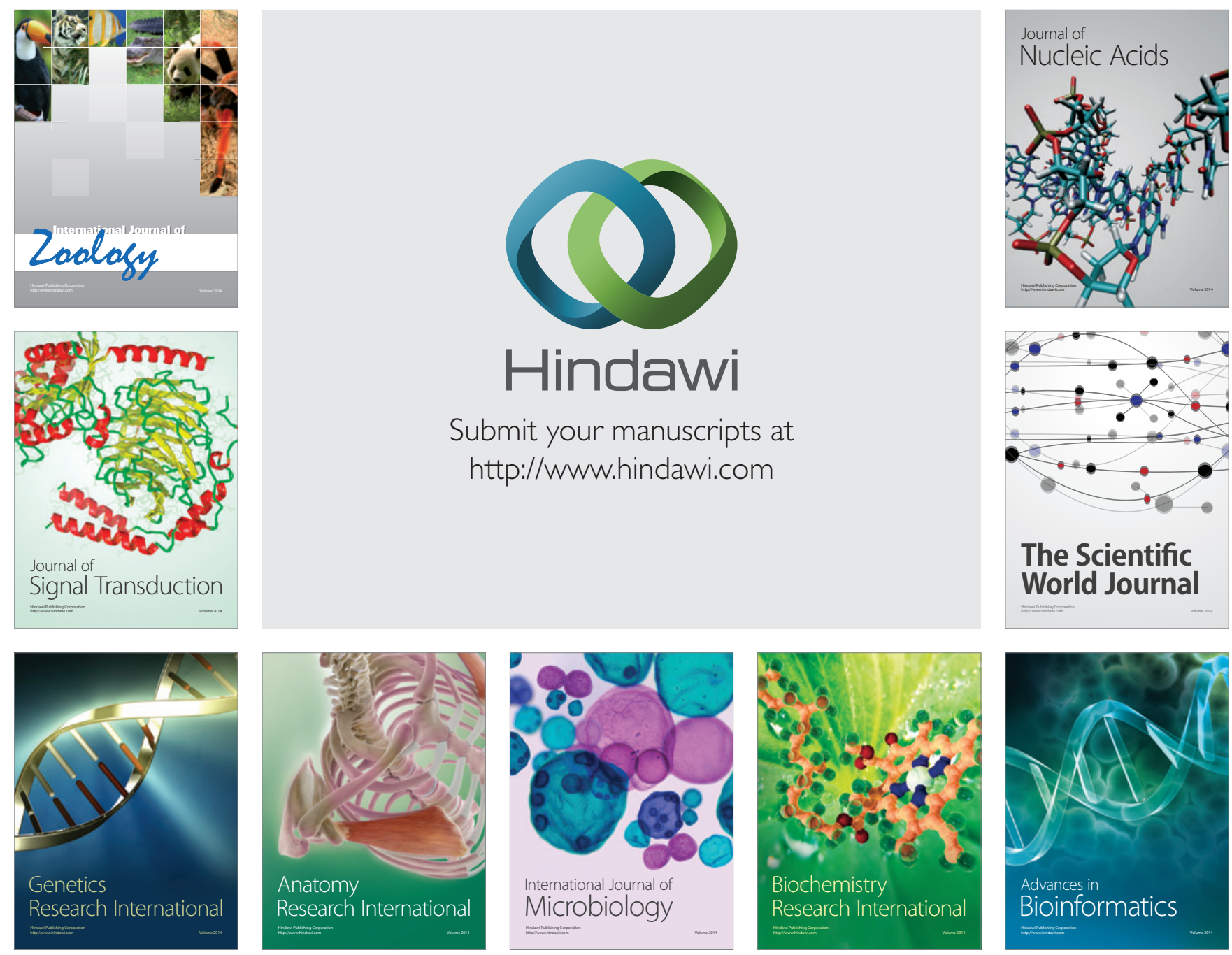

The Scientific World Journal
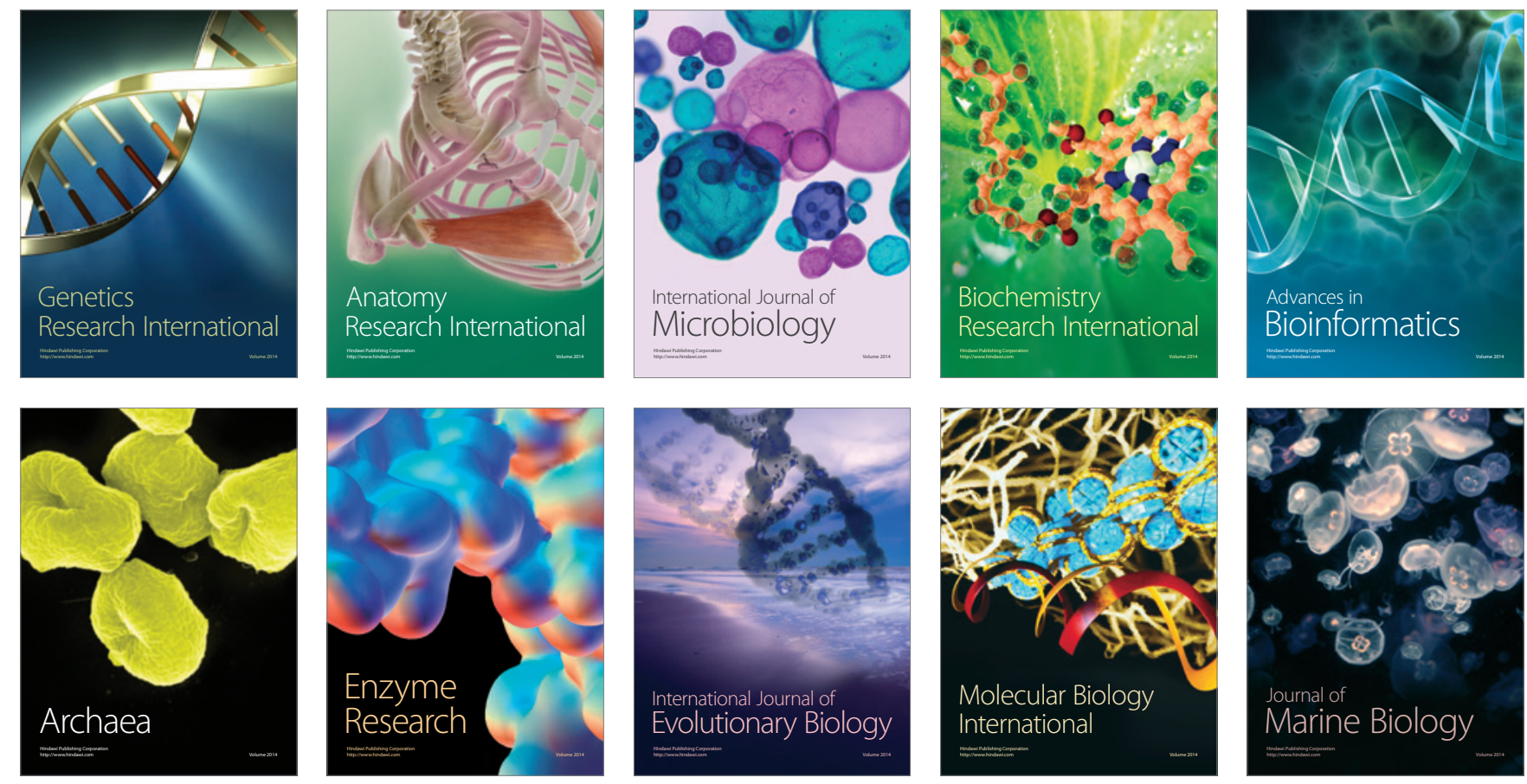